TITLE:

\title{
Higher breast cancer conspicuity on dbPET compared to WB- PET/CT(Abstract_要旨)
}

$\operatorname{AUTHOR}(S)$ :

Nishimatsu, Kayo

\section{CITATION:}

Nishimatsu, Kayo. Higher breast cancer conspicuity on dbPET compared to WB-PET/CT. 京都大学, 2017, 博士(医学)

ISSUE DATE:

2017-07-24

URL:

https://doi.org/10.14989/doctor.k20612

\section{RIGHT:}

Kayo Nishimatsu, Yuji Nakamoto, Kanae K. Miyake, Takayoshi Ishimori, Shotaro Kanao, Masakazu Toi, Kaori Togashi, Higher breast cancer conspicuity on dbPET compared to WB-PET/CT, European Journal of Radiology, Volume 90, 2017, Pages 138-145, ISSN 0720048X,

http://dx.doi.org/10.1016/j.ejrad.2017.02.046.(http://www.sciencedirect.com/science/arti cle/pii/S0720048X17301006) 
